\title{
Territoire et espace public dans l'Union européenne : les dimensions internes et externes (Partie 2)
}

Andy SMITH et Jean-Louis Quermonne

\section{CpenEdition}

Journals

Édition électronique

URL : http://journals.openedition.org/conflits/631

DOI : $10.4000 /$ conflits.631

ISSN : $1777-5345$

Éditeur :

CCLS - Centre d'études sur les conflits lilberté et sécurité, L'Harmattan

Édition imprimée

Date de publication : 15 mai 1996

ISSN : 1157-996X

Référence électronique

Andy SMITH et Jean-Louis Quermonne, «Territoire et espace public dans l'Union européenne : les dimensions internes et externes (Partie 2) », Cultures \& Conflits [En ligne], 21-22 | printemps-été 1996, mis en ligne le 15 mars 2006, consulté le 30 mars 2021. URL : http://journals.openedition.org/conflits/ 631 ; DOl : https://doi.org/10.4000/conflits.631

Ce document a été généré automatiquement le 30 mars 2021

Creative Commons License 


\title{
Territoire et espace public dans l'Union européenne : les dimensions internes et externes (Partie 2)
}

\author{
Andy SMITH et Jean-Louis Quermonne
}

1 L'Union européenne et les mutations territoriales en dehors de ses frontières

2 Jusqu'à la signature de l'Acte Unique européen, en 1986, la coopération politique extérieure des États membres de la Communauté Économique Européenne n'a pas fait l'objet d'un traité. Elle a seulement résulté de l'approbation par le Conseil Européen d'une série de rapports commandités par celui-ci, dont, le plus célèbre, le rapport Davignon, approuvé le 27 octobre 1970, comportait l'engagement pour les gouvernements: " d'assurer par une information et des consultations régulières une meilleure compréhension mutuelle sur les grands problèmes de politique internationale ; de renforcer leur solidarité en favorisant une harmonisation des points de vue, la concertation des attitudes et, lorsque cela parait possible et souhaitable, des actions communes ".

De la coopération politique extérieure...

4 Sur cette base, cependant, précisée et complétée par d'autres textes, un certain nombre de positions communes exprimant le pont de vue de la CEE ont été adoptées qui permettent de formuler l'hypothèse d'une convergence des États en vue d'assurer la stabilité des relations internationales et de promouvoir la paix dans le monde. Rappelons quelques unes de ces positions. Elles ont notamment concerné : La solidarité manifestée par les États membres de la CEE à l'égard du Royaume-Uni lors du conflit des Malouines en 1982, en vue du rétablissement du statu-quo territorial. Les prises de position successives du Conseil Européen concernant la situation conflictuelle au Moyen-Orient (notamment la Déclaration de Venise du 13 juin 1980), le problème libanais, la guerre Iran-Irak, le dialogue Nord-Sud, les tensions en Afrique australe, la crise afghane et la crise polonaise (la position des Neuf ayant été accompagnée, dans ce dernier cas, par la livraison de 400000 tonnes de produits alimentaires).

- Enfin et surtout l'affirmation de l'unité et de l'influence des pays membres de la 
Communauté Européenne comme leader du groupe occidental lors de la Conférence d'Helsinski, de 1971 à 1975, et de la création de la C.S.C.E., devenue aujourd'hui l'O.S.C.E. ${ }^{1}$.

5 ...A la politique étrangère et de sécurité commune Dans ces différents cas de figure, l'action menée par la Communauté, sans pour autant renier le Droit des peuples à disposer d'eux-mêmes, a incontestablement joué en faveur de la stabilité des frontières. Et c'est cette même politique qu'a poursuivi l'Union européenne en prenant à son compte l'initiative française incitant les pays d'Europe centrale et orientale à conclure un Pacte de Stabilité garantissant, en même temps que le respect des frontières, le sort des minorités nationales qui s'y trouvent incluses, et en mesurant quelques actions communes en faveur de la paix ${ }^{2}$. Malheureusement, le no man's land qui a séparé la chute du Mur de Berlin en 1990 de la ratification du Traité de Maastricht le 1er novembre 1993 n'a pas permis de donner à cette action la continuité et surtout la fermeté qu'aurait nécessité le règlement de la crise yougoslave. Et la valse-hésitation qui s'est produite entre le Principe des nationalités et le respect des frontières a servi de toile de fond à la contradiction qui a opposé l'Allemagne d'une part, la France et la Grande-Bretagne d'autre part quant au sort des anciennes républiques fédérées de Yougoslavie. Aussi, l'Union européenne en voie de gestation n'a-t-elle pas été à la hauteur du conflit. Et l'appel adressé tant aux États-Unis qu'à l'O.N.U. a témoigné de l'incapacité de l'Union européenne à mettre en œuvre la politique étrangère et de sécurité prévue dans le cadre du deuxième pilier du traité de Maastricht. Cependant, c'est le souci de garantir ou de promouvoir la stabilité en Europe qui a conduit plusieurs États membres de l'Union européenne à mettre à la disposition de l'O.N.U. d'importants contingents de " casques bleus ", à apporter une aide alimentaire et humanitaire à la Bosnie, à organiser une série de conférences internationales destinées à apporter la paix et à participer au " groupe de contact " qui vise au même but. Mais, dans le même temps, le conflit yougoslave a révélé à l'Occident la profondeur des tensions existantes dans l'Europe balkanique et la faible capacité des organisations de sécurité collective insuffisamment structurées, telle l'O.S.C.E., à contribuer de manière efficace au règlement de conflits dont les racines remontent à plusieurs siècles. A l'inverse, cette conjoncture a permis de vérifier la portée de la réconciliation réalisée entre l'Allemagne et la France depuis la Déclaration Schuman du 9 mai 1950 évitant que leurs divergences momentanées ne dégénèrent en conflit. Cette leçon ne semble pas avoir été perdue par les gouvernements des pays d'Europe centrale et orientale qui réclament leur intégration la plus rapide possible à l'Union européenne et à l'O.T.A.N. L'Union européenne étant entrée seulement en vigueur le 1er novembre 1993, il est prématuré de vouloir juger de l'impact que pourrait exercer la politique étrangère et de sécurité commune qui a remplacé la coopération politique extérieure sur les mutations territoriales en Europe. Notons toutefois la continuité qui caractérise l'attitude observée par la C.E.E., à l'initiative de Jacques Delors, au lendemain de la chute du mur de Berlin, à la fois pour associer celle-ci à l'action du chancelier Kohl en vue de réaliser l'unification allemande et pour apporter aux pays de l'Europe centrale et orientale, libérés de la domination soviétique un appui décisif. Sans doute, parce que la communauté n'était encore qu'économique, le programme Phare n'a-t-il pas eu le retentissement $d u$ plan Marshall. Cependant, n'ayons garde d'oublier que l'aide communautaire aux pays de l'Est représente $75 \%$ des contributions occidentales et que dès l'origine les pays du G7 suivis par ceux du G24 ont reconnu le rôle pionnier joué par la Commission européenne en lui confiant le soin de coordonner cette aide. C'est dans 
l'axe de cette continuité, tendant à promouvoir à la fois la sécurité, la démocratie et l'économie de marché, que l'Union européenne amorce aujourd'hui une politique étrangère et de sécurité commune, encore insuffisamment cohérente, mais néanmoins présente. Deux exemples suffiront à illustrer les tentatives entreprises en vue d'assurer à l'Est et au Sud de l'Europe, la conciliation entre le Droit des peuples à disposer d'euxmêmes et la sécurité des frontières, afin de promouvoir un espace européen pacifié. Le premier concerne l'apaisement des tensions entre la Grèce et la Turquie. Réunis le 6 février 1995 à Bruxelles, les ministres des affaires étrangères des Quinze sont parvenus à un accord de principe ouvrant la voie à une union douanière avec la Turquie et permettant à terme l'ouverture de pourparlers avec Chypre en vue de l'adhésion de l'île à l'Union européenne. En compensation de cette union douanière qui aurait dû intervenir le 1er janvier 1996, l'Union européenne s'engage à ouvrir des négociations d'adhésion avec Chypre six mois après la conclusion de la Conférence intergouvernementale de 1996. Mais malgré les concessions consenties tardivement par le gouvernement grec, la conjoncture politique intérieure de la Turquie risque de conduire le Parlement européen à retarder de donner son avis conforme à l'accord d'Union douanière conclu avec ce pays. Le cas d'espèce illustre, à la fois, la difficulté de résoudre par voie diplomatique des problèmes ancestraux et celle de mener une politique étrangère et de sécurité commune effective tant que sera maintenu le principe du vote à l'unanimité au Conseil de l'Union. Le second exemple concerne également l'Europe centrale et orientale. Mais il est d'une plus grande envergure puisqu'il concerne l'association et l'éventuelle adhésion des pays membres de cette région à l'Union européenne. En fait, l'enjeu n'est autre que celui de l'établissement des frontières Est de l'Europe. Or, chacun sait que si celles-ci lui ont été imposées au long de la " guerre froide " par l'existence du rideau de fer, la destruction de celui-ci lui a brutalement ouvert la possibilité de les déterminer plus librement. Lourde liberté à assumer! Car il s'agit de l'exercer dans une conjoncture où le " retournement du monde ", selon l'expression de Bertrand Badie et de Marie-Claude Smouts ${ }^{3}$. Mais une telle orientation, destinée à façonner progressivement l'Europe à 25 ou à 30 États, doit être accompagnée parallèlement d'une politique de partenariat effective avec la Russie et les autres pays membres de la Communauté des États Indépendants. En effet, l'adhésion à l'Union européenne ne sera rendue possible que par une étroite coopération entre celle-ci et la C.E.I. et, tout particulièrement la Russie. Si non, l'attitude de cette dernière à l'égard de l'Union européenne et de l'Union de l'Europe occidentale risquerait fort de ressembler à celle que son ministre des affaires étrangères a cru devoir prendre à l'égard d'une éventuelle adhésion des PECO à l'O.T.A.N. De ce point de vue comme en d'autres, l'affirmation d'une Europe indépendante par rapport aux États-Unis d'Amérique apparaît comme l'une des clés de son élargissement. Ce qui ne signifie pas, bien au contraire, que du côté atlantique un autre partenariat ne doive pas s'établir entre l'Union européenne et les Américains. Il semble bien, par conséquent, que contrairement à la perspective dessinée par les " visionnaires " qui préconisent une Europe allant de Vancouver à Vladivostok, la reconstruction d'un nouvel ordre territorial dans l'hémisphère nord passe par la constitution de trois ensembles entre lesquels un partenariat effectif pourrait être institué. Dans le cadre de ce partenariat, l'Union européenne occuperait une position centrale (du moins au regard des cartes géographiques auxquelles nous sommes habitués) qui aurait pour frontières à l'Ouest, l'Océan Atlantique, au sud la Méditerranée et à l'Est, les limites de la C.E.I. Les points éventuellement litigieux à l'Est 
pourraient concerner les États baltes, dont les Occidentaux n'ont jamais reconnu l'annexion avant-guerre par l'Union Soviétique mais où vivent d'importantes minorités russophones, et au Sud-Est la Turquie dont la question de savoir si elle doit être ou non intégrée à l'Union européenne semble encore en suspens. Mais à l'intérieur de ses nouvelles frontières, l'Union européenne n'en aurait pas moins un autre problème à régler : celui du statut des minorités inclues dans les limites intérieures d'une partie de ses États. Or si les tensions en Irlande du Nord, dans le Pays-Basque ou en Corse sont peut être en voie de s'apaiser, qu'en sera-t-il demain en Europe Orientale et dans les Balkans? Ne faudra-t-il pas alors inventer des solutions politiques tendant à dissocier les nationalités des territoires, selon la formule préconisée au début du siècle par Karl Renner à l'intention de l'Autriche-Hongrie. Rappelons que cette formule visait à substituer au couple infernal autodétermination des peuples - respect des frontières, l'autodétermination de chaque citoyen envers des nationalités dégagées de toute emprise territoriale ? ${ }^{4}$ Reste à se demander si l'espace ainsi délimité à l'intérieur de l'Union européenne pourra être considéré comme un espace public? Telle est la troisième question que ce chapitre a pour mission d'aborder.

6 L'Union européenne génératrice d'un nouvel espace public? L'espace protégé par le tarif extérieur du Marché unique européen est bel et bien un espace économique. A l'intérieur de celui-ci s'exercent, en effet, les quatre libertés prescrites par le Traité de Rome et confortées par l'Acte Unique: la libre circulation des marchandises, des services, des capitaux et des personnes. Encore que pour ces dernières des restrictions subsistent, que la mise en oeuvre effective des accords de Schengen ne devrait pouvoir lever que sur une partie du territoire de l'Union européenne. Quant à l'existence d'un espace public, elle fait naturellement problème si l'on entend par là, sous l'inspiration d'Habermas, un espace sur lequel s'exercent pleinement, au bénéfice de ses ressortissants, les allégeances et les droits qui sont généralement attachés à la citoyenneté car pour l'auteur, l'espace public n'est pas seulement une sphère où circule la communication, mais également une scène politique où s'exprime la délibération ${ }^{5}$. Or il n'apparaît pas - ou pas encore - que l'Union européenne constitue cette scène.

7 D'un espace de politiques publiques intégré... Dans l'immédiat, il résulte de l'application des traités de Rome et de Maastricht que ses frontières extérieures dessinent un territoire sur lequel s'entrecroisent des politiques publiques: les unes communautaires, les autres nationales ${ }^{6}$. Relevant de moins en moins des compétences exclusives soit de la Communauté, soit des États membres, ces politiques ont de plus en plus tendance à se développer sur un espace ressortissant à leurs compétences concurrentes. Pendant plusieurs décennies, son extension a été imputée à un phénomène d'engrenage, qualifié par les auteurs anglo-américains de spill over, et juridiquement fondé sur l'existence de pouvoirs implicites reconnus à l'article $235 \mathrm{du}$ Traité CEE. Depuis le Traité de Maastricht, l'élargissement du champ d'intervention de la compétence concurrente de l'Union européenne et des États résulte de l'inventaire qu'en donne l'article G. Mais le Traité en fixe en même temps les limites en instituant le principe de subsidiarité. Il résulte que le " no man's land " ainsi élargi entre les actions publiques que se partagent la Communauté et les États, a ouvert un espace considérable à la coopération intergouvernementale, qu'elle s'exerce de manière intégrée au sein du Conseil de l'Union, ou qu'elle se pratique en marge des instances communautaires sous forme diplomatique. Si bien que des auteurs familiers du fédéralisme coopératif, tel qu'il se pratique au Canada, en Allemagne ou en Suisse, s'interrogent. Ils se demandent, en effet, si l'on est pas en train d'assister à la naissance, dans le cadre de l'Union 
européenne, d'un " fédéralisme intergouvernemental " que refuseraient encore de reconnaître à la fois ceux qui rejettent toute référence à la vocation fédérale de l'Union et ceux pour lesquels l'État fédéral est la seule voie légitime qui puisse s'ouvrir à elle7. ... à la virtualité d'une espace public diversifié Quoi qu'il en soit, un tel espace de politiques publiques ne s'identifie pas nécessairement à un espace public s'il n'offre pas au citoyen la possibilité de participer à leur élaboration dans les conditions que lui offre généralement la mise en oeuvre de la démocratie ${ }^{8}$. Or, est-il besoin de rappeler ici les critiques dont le système politique de la Communauté européenne a depuis longtemps fait l'objet de la part de ceux qui voient en lui une illustration regrettable de la technocratie? Peu importe que celle-ci résulte davantage des pratiques intergouvernementales génératrices de " comitologie " que de la Commission européenne, composée depuis longtemps de politiques plus que de fonctionnaires ${ }^{9}$. L'essentiel tient au " déficit démocratique " résultant, selon les uns de la faiblesse des pouvoirs du Parlement européen et selon les autres de la mise à l'écart des parlements nationaux, mais dans les deux cas de la carence du pouvoir de délibération. Or la crise de la représentation est générale en Europe et dans le Monde, ce qui confère un peu partout aux groupes d'intérêt et de pression un rôle de médiation dont le caractère démocratique est encore loin d'être reconnu, et ne saurait constituer un substitut légitime à l'absence de délibération. Sans doute, à l'initiative du gouvernement espagnol, le Traité de Maastricht a-t-il institué, sous le nom de citoyenneté européenne, une citoyenneté de superposition qui, sans altérer en quoique ce soit l'allégeance de ses bénéficiaires à leurs États membres respectifs, confère à ceux-ci un minimum de droits supplémentaires. Et, dès lors, la Communauté européenne n'apparaît plus exclusivement comme une société d'États et d'opérateurs économiques, mais comme un espace politique ouvert - ou du moins entr'ouvert - à l'expression des citoyens. Mais cette nouvelle dimension de l'Union européenne est encore assez peu perceptible. Et certains États s'efforcent encore de l'occulter en minimisant la portée de l'électorat et de l'éligibilité des ressortissants communautaires sur leur territoire lors des élections au Parlement Européen et aux conseils municipaux. En outre, quarante-cinq ans de construction européenne n'ont pas provoqué, comme l'avaient escompté ses " pères fondateurs " l'émergence d'un État fédéral européen. Et la tentative entreprise par les chrétiens-démocrates allemands pour équilibrer l'élargissement de l'Union européenne à l'Est par la constitution d'un " noyau " de structure fédérale, afin d'éviter à celle-ci de dégénérer en zone de libre échange, fait encore pousser les hauts cris. Par conséquent, il serait aujourd'hui illégitime de vouloir comparer l'espace circonscrit à l'intérieur du marché unique européen avec celui d'un État. " Un Zollverein n'est pas une patrie ", peut s'exclamer Jean Leca ${ }^{10}$. Se trouve, dès lors, posée la question de savoir s'il y a place, en marge des États, à des espaces publics diversifiés où puisse progressivement s'épanouir la démocratie ? La question affecte d'ailleurs à la fois le niveau transnational et le niveau infra-étatique. Et elle se trouve, parfois, dramatisée par la revendication du monopole d'un espace démocratique légitime au profit de l'État-nation. Or, sans rouvrir un débat largement engagé ${ }^{11}$, observons simplement la double mutation que subissent, depuis un demi-siècle au moins, des instances administratives qui tendent de plus en plus à devenir politiques. Cette observation vaut à la fois pour les collectivités territoriales, communales et régionales, et pour les organisations internationales, dont beaucoup se dotent, à côté d'institutions intergouvernementales, d'assemblées parlementaires de nature consultative. Par conséquent, la dimension politique s'étendant à la faveur de la décentralisation et de la mondialisation à de multiples 
niveaux, l'on ne saurait s'étonner de l'apparition d'espaces publics qui ne forment pas, pour autant, des États. Leur degré de démocratie varie considérablement d'institution à institution. La première partie de ce rapport montre que, face à la politique régionale de la Communauté européenne, le partenaire émergeant au sein des États membres peut être tantôt de nature administrative, comme au Royaume-Uni, ou tantôt de nature politique, comme dans le cas des Communautés autonomes espagnoles. Et c'est alors seulement que la démocratie de proximité pénètre les rouages du processus de décision communautaire et vise à amorcer, encore timidement, comme dans le cas du " quadrige européen " associant Rhône-Alpes à la Catalogne, à la Lombardie et au BadeWurtemberg, un mode de relation transnational entre les régions ${ }^{12}$. L'on pourrait, parallèlement, démontrer que son degré d'intensité varie au plan mondial du Mercosul à l'Alena ou de l'A.F.T.A. à l'A.P.E.C., sans oublier l'Afrique de l'ouest, selon le stade d'intégration en cours de réalisation à l'échelle des continents. De ce point de vue, le " modèle " formé par l'Union européenne semble bien le plus intensément engagé sur la voie de l'union politique sinon sur celle de la démocratie. Ce qui relativise les critiques relatives à la portée de son propre " déficit ", mais ne permet pas d'observer encore sauf peut-être entre les pays membres des accords de Visegrad ou du Mercosul - un phénomène de mimétisme évident. Or tandis que la mondialisation conduit à restreindre la souveraineté effective des États et alors que la fin de la guerre froide tendait à modérer les tensions ${ }^{13}$, le penchant auquel succombent trop de nations, d'ethnies ou de minorités est le recours à la violence, tantôt sous forme de guerre étrangère limitée, tantôt sous forme de guerre civile, tantôt encore sous forme de terrorisme. Et il en résulte des conflits que la communauté internationale n'est pas apte à maîtriser. Ce réveil des nationalismes tend à opposer, de façon ouverte ou de manière latente, à la lente formation du " village-monde ", un mouvement de repli aux accents racistes ou guerriers. Bref, la restructuration des territoires réalisée dans un mouvement centrifuge grâce au libre échange, ne pourra jamais conjurer les risques de déstructuration des territoires causés par la recherche des identités. Sans même revenir sur la lancinante guerre yougoslave, l'on est obligé d'observer en effet qu'à l'intérieur même de l'Union européenne, les tensions violentes existant en Irlande du Nord, au Pays Basque ou en Corse n'ont pas encore été complètement apaisées. Et à l'échelle de la " Grande Europe ", pour un divorce réalisé à l'amiable entre la République Tchèque et la Slovaquie, combien de foyers de tension subsistent, qui risquent toujours d'enflammer les nationalités et les minorités. Conclusion Au moment de conclure, il paraît difficile d'observer en Europe, face au bouleversement du monde provoqué par les effets en chaîne de la chute du mur de Berlin, un nouvel ordre territorial susceptible d'offrir une lecture claire des espaces publics en voie de gestation. S'agissant plus précisément de l'Union européenne, l'on continue à s'interroger sur la profondeur des changements intervenus depuis la fin de $1989^{14}$. Or, à côté de la flexibilité qui semble favoriser la restructuration des espaces économiques tels que celui de Visegrad, dans une vaste zone située entre l'Union européenne et la Russie, l'on observe la persistance de tendances lourdes à l'éclatement, héritées du passé, y compris de la conquête turque de l'Europe balkanique et de la coupure entre les églises d'orient et d'occident. L'on pourrait en dire autant de la dialectique opposant les mouvements d'intégration en voie de formation (de l'unification allemande au processus de pré-adhésion à l'Union européenne) et les tendances au séparatisme, dont le divorce tchéco-slovaque en marge de l'Union européenne paraît avoir pour symétrie à l'intérieur de celle-ci les tensions qui affectent la Belgique, sans 
parler de celles qui agitent l'Italie et justifiant que l'on traite, encore aujourd'hui, des séparatismes en Europe ${ }^{15}$. Il n'en reste pas moins vrai que le bilan des quarante cinq années qui nous éloignent de l'acte fondateur de la construction européenne, à savoir la Déclaration Schuman du 9 mai 1950 témoigne largement en faveur de la stabilité réalisée entre les six pays fondateurs de l'aventure communautaire, devenus progressivement 9, puis 10, puis 12 et aujourd'hui 15 États étroitement associés. Il n'est donc pas utopique d'escompter que la perspective de l'élargissement de l'actuelle Union européenne à 20 , à 25 ou à 30 , favorisera à terme la même stabilité et ouvrira la voie, dans un même mouvement, à un véritable partenariat pour la paix tant avec la Russie et les autres pays de la Communauté des États indépendants, qu'avec l'Amérique du Nord et du Sud et l'Afrique, sans oublier naturellement le Moyen-Orient et l'Asie. Encore est-il que l'effet de contagion - si non de mimétisme - ne pourra s'affirmer que si la Grande Europe, loin de se diluer dans une simple zone de libre échange, sait transformer l'espace économique qu'elle est appelée à former en un espace public structuré. D'où l'importance que revêt l'effort de réflexion entrepris pour concilier l'inéluctable géométrie variable qui affectera son architecture avec le renforcement d'une pôle attractif, qu'on l'appelle ou non " noyau dur ", seul capable de servir de clé de voûte à un ensemble qui risquerait sans cela au mieux de se désagréger et au pire d'éclater.

Revenir à la partie précédente

\section{NOTES}

1. Ces différentes prises de position ont été analysées par Béatrice Taulegne dans son ouvrage sur le Conseil Européen, PUF, 1993, p. 343 à 397.

2. Sur ces quelques actions communes, l'on pourra se reporter à l'ouvrage de Fabrice Fries, Les grands débats européens, Paris, Le Seuil, 1995, p. 103.

3. Bertrand Badie, Marie-Claude Smouts, Le retournement, n'offre aux restructurations territoriales en Europe qu'une flexibilité limitée. Alors même que la restauration d'une éventuelle " glaciation " à l'Est rendrait, à nouveau, la coupure entre les deux Europes inéluctable, l'impatience des gouvernements des PECO s'explique face au souci de leurs homologues occidentaux de " donner du temps au temps ". Confrontés à cette situation, les Conseils européens successifs, notamment celui réuni en décembre 1994 à Essen sous présidence allemande, ont déclenché en direction des pays de l'Europe centrale et orientale (les PECO) un processus de pré-adhésion, qu'avaient déjà largement esquissé les accords européens d'association[[Françoise de La Serre, Christian Lequesne, Jacques Rupnik, L'Union Européenne : ouverture à l'Est ?, PUF, 1994.

4. Stéphane Pierré-Caps, " Karl Renner et l'État multinational . Contribution juridique à la solution d'imbroglios politiques contemporains, Droit et Société ", Revue Internationale de Droit et de Sociologie juridique, L.G.D.I., n 27, 1994. Du même auteur : La multination, l'avenir des minorités en Europe centrale et orientale, Odile Jacob, 1995. Alain Fenet et al., Le droit et les minorités, analyses et textes, Bruyland, 1995. 
5. Jürgen Habermas, L'espace public, Payot, 1993. Cf. également Bernard Manin, " Volonté générale ou délibération ? Esquisse d'une théorie de la délibération politique ", Le Débat, $n^{\circ}$ 33, janvier 1985.

6. Cf. en particulier : Meny, Muller, Quermonne (dir.), Les Politiques publiques en Europe, l'Harmattan, 1995 ; G. Druesne, Droit et Politiques de la Communauté et de l'Union Européenne, 3ème éd., P.U.F., 1995 ; J.C. Leygues, Les Politiques internes de l'Union Européenne, L.G.D.J., 1995 ; B. Jobert (dir.), Le Tournant néo-libéral en Europe, L'Harmattan, 1994 ; Mireille Pongy, Guy Saez, Politiques culturelles et régions en Europe, L'Harmattan, 1994.

7. Maurice Croisat, Le fédéralisme dans les démocraties contemporaines, 2ème éd., Clefs, Montchrestien, 1995.

8. Marc Abélès, " A la recherche d'un espace public communautaire ", Pouvoirs, $n^{\circ}$ 69, avril 1994, p. 117 et sq.

9. Jean-Louis Quermonne, " Le spectre de la technocratie et le retour du politique ", Pouvoirs, n 69, avril 1994, p. 7.

10. Jean Leca, " Après Maastricht, sur la prétendue résurgence du nationalisme ", Témoin, n 1, décembre 1993, p. 37.

11. Citons sur ce point : Jean-Marc Ferry, Paul Thibaud, Discussion sur l'Europe, Calmann-Lévy, 1992 ; Dominique Schnapper, La Communauté des Citoyens, sur l'idée moderne de nation, Gallimard, 1994 ; Jacques Rupnik (dir.), Le déchirement des nations, Paris, Le Seuil, 1995.

12. Cf. le rapport présenté par Pierre Kukawka dans le cadre du colloque sur les régions et l'Europe organisé les 4, 5 et 6 octobre 1995 à l'Institut d'études politiques de Rennes. 13. Cf. sur ce point le beau livre de Pierre Grosser, Les temps de la guerre froide, Ed. Complexe, 1995, notamment p. 335 et sq.

14. Tel a été le cas, lors d'une réunion organisée par le groupe de travail sur les relations internationales animé par Marcel Merle, dans le cadre de l'A.F.S.P. en 1995. 15. Christian Bidegaray (dir.), Les séparatismes en Europe, travaux du Colloque de Nice, à paraître chez Economica.

\section{INDEX}

Index géographique : Union européenne

Mots-clés : territoire(s) et territorialité, construction européenne, droit 